\title{
Grazing-angle characterization of photosynthetic oxygen evolution protein monolayers
}

\author{
Eileen Y. Yu \\ Biophysics Research Division, University of Michigan, Ann Arbor, MI 48109 \\ James E. Penner-Hahn \\ Department of Chemistry, University of Michigan, Ann Arbor, MI 48109 \\ Charles F. Yocum \\ Department of Biology, University of Michigan, Ann Arbor, MI 48109 \\ Robert H. Mayer and Ingrid J. Pickering \\ Stanford Synchrotron Radiation Laboratory, Stanford, CA 94309
}

(Presented on 18 October 1995)

\begin{abstract}
Variable-period x-ray standing wave (XSW) spectroscopy has been shown to be a practical probe for studying metalloproteins. The photosynthetic oxygen evolving complex (OEC) is a transmembrane multipolypeptide complex that catalyzes the oxidation of water to dioxygen. The OEC contains Mn, $\mathrm{Ca}$, and $\mathrm{Cl}$ and is potentially amenable to study by XSW. In this feasibility study, preliminary results on OEC samples deposited on Au mirrors are discussed. First XSW measurements from the SSRL grazing-incidence setup are presented. () 1996 American Institute of Physics.
\end{abstract}

\section{INTRODUCTION}

The x-ray standing wave (XSW) technique has proven to be a unique and precise tool for depth profiling of heavy atoms in thin films and on surfaces since its first application thirty years ago. ${ }^{1}$ Recent advances in generating $\mathrm{x}$-ray standing waves with long $^{2}$ and variable periods have prompted applications of the XSW method to biologically relevant systems. In particular, it has been demonstrated that XSW measurements are a powerful probe for determining heavy atom distributions within organic macromolecular structures with angstrom-level precision, ${ }^{3}$ both in membranes ${ }^{4}$ and in metalloproteins. ${ }^{5}$ Since XSW provides information on the position and the width of heavy atom layer distributions as a function of the distance normal to the sample surface, measurements on an oriented monolayer of a metal-containing protein can yield useful information on the molecular structure of the native protein. A metalloprotein complex of great interest is photosystem II (PSII), which is a Mncontaining component of oxygen photosynthetic electron transfer chains.

The oxygen evolving complex (OEC) in PSII is the catalytic unit responsible for water oxidation and the concomitant release of oxygen molecules. Its active site is a cluster of four Mn atoms that functions as the source of oxidizing equivalents in the electron transfer process. Biochemical manipulations in conjunction with conventional spectroscopies, such as EPR, have revealed some key aspects of the oxygen evolution process, including a requirement for cofactors such as $\mathrm{Cl}^{-}$and $\mathrm{Ca}^{2+}$ for OEC activity. ${ }^{6}$ Extended $\mathrm{x}$-ray absorption fine structure spectroscopy (EXAFS) has provided basic radial structure information. Nevertheless, many structural details of the OEC remain elusive. In combination with a well-characterized oriented sample, XSW as a highly sensitive directional probe offers the most direct determination of the relative positions of cofactors (e.g., $\left.\mathrm{Ca}^{2+}, \mathrm{Cl}^{-}\right)$and the $\mathrm{Mn}$ atoms within the cluster.

In this study, the feasibility of making XSW measurements on an oriented OEC monolayer has been investigated by the generation of a variable period XSW under specular reflection conditions. The inherent sensitivity of the element-specific XSW probe is demonstrated to be in good agreement with our calculations, showing that it is possible to obtain statistically significant signals from a sample with low heavy atom density, such as a monolayer of PSII. Preliminary XSW and reflectivity results, as well as ellipsometry data, suggest that we have successfully deposited the Mn-containing protein onto a gold mirror utilizing the well-characterized phenomenon of self-assembled monolayer (SAM) formation.

\section{XSW DURING SPECULAR REFLECTION}

$\mathrm{X}$-rays undergo total external reflection when impinging on an optical boundary (a mirror) at an angle less than the critical angle since the index of refraction is less than unity for $\mathrm{x}$-rays in matter. Coherent interference between the incident wave and the specularly reflected wave results in the generation of a standing wave above the mirror surface; this phenomenon is essentially a zeroth-order Bragg diffraction condition with the mirror surface as the diffraction plane and an infinite $d$ spacing. At $\theta=0$, the first antinode of the XSW is infinitely far above the mirror surface. As the incident angle $\theta$ is increased, the first antinode shifts inward until it coincides with the mirror surface at the critical angle $\theta_{\mathrm{c}}$ while the other antinodes compress inward (much like a collapsing bellows) with decreasing period, $D$, given by $D=\lambda /(2 \sin \theta)$, where $\lambda$ is the wavelength of the incident beam. At incident angles greater than the critical angle, the specularly generated XSW quickly disappears as the bulk of the incident wave is transmitted rather than reflected. ${ }^{7}$ An XSW electric field generated in this way has been shown to be well defined up to $1,000 \AA$ above the mirror surface. ${ }^{8}$

During the specular-reflection conditions as the antinodes move inward, the magnitude of the electric field at a fixed distance above the mirror surface undergoes smooth transitions from maxima to minima in an oscillatory manner. Since intensity varies as the square of the magnitude of the electric field, there is a four-fold increase in the intensity of the XSW resulting from the superposition of the two 
interfering waves. For heavy atoms situated at a fixed distance from the mirror surface, characteristic modulations appear in the x-ray fluorescence yield as a result of the photoelectric effect. Since the probability of excitation is proportional to the intensity of the electric field at the center of the heavy atom, a maximum in fluorescence intensity will be observed when an antinode is in the plane of the heavy atoms and a minimum will be observed when a node coincides with the heavy atom layer. The distance at which the atoms are displaced from the mirror surface can be determined by monitoring the reflectivity and fluorescence yields simultaneously. ${ }^{3}$

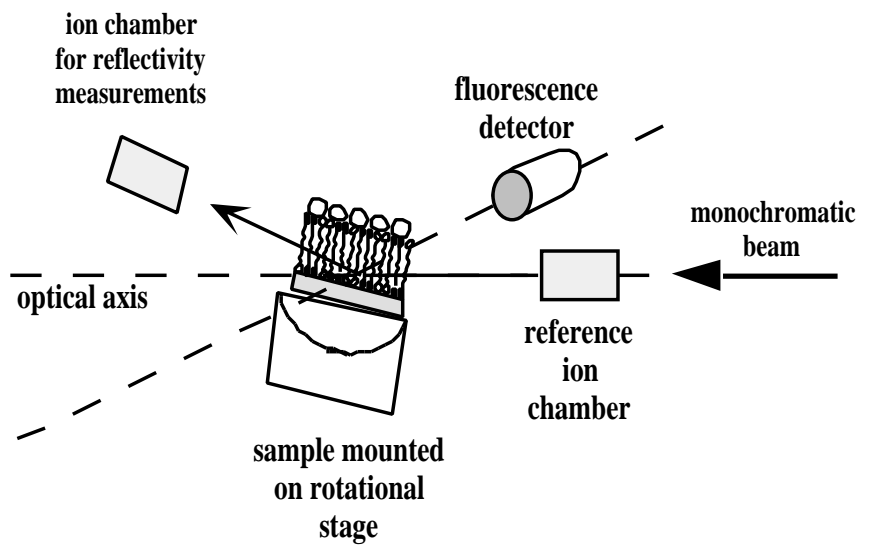

FIG. 1. Experimental setup for XSW measurements.

\section{EXPERIMENT}

Oriented monolayers of PSII core complexes enriched in OEC were deposited onto the positively charged surface of a sputtered gold substrate that had been treated with amineterminated thiol molecules $\mathrm{SH}\left(\mathrm{CH}_{2}\right)_{2} \mathrm{NH}_{2}$. The thiol-coated $\mathrm{Au}$ mirrors were incubated overnight on ice in solutions of OEC $(135 \mathrm{mg} / \mathrm{ml} \mathrm{Chl})$ with the detergent $\mathrm{n}$-dodecyl- $\beta$-Dmaltoside (DM) or in suspensions of OEC without detergent. All solutions were buffered at $\mathrm{pH}$ 6. Once removed from solution, the samples were rinsed in five consecutive buffer baths and dried with a gentle stream of $\mathrm{N}_{2}$ gas. Formation of SAMs and subsequent ellipsometry measurements were performed under clean room conditions at the Solid State Electronics Laboratory at the University of Michigan. Parallel samples prepared without the use of the tethering thiol molecules were also characterized.

$\mathrm{XSW}$ data were collected with the incident energy fixed at $10 \mathrm{keV}$, well away from the relevant atomic absorption edges. A general schematic diagram is shown in Figure 1. Multichannel analyzer (MCA) data were collected at incidence angles around $6 \mathrm{mrad}$ in a region of constant reflectivity below the critical angle of Au. All x-ray measurements were taken at the grazing incident spectrometer at the Stanford Synchrotron Radiation Laboratory (SSRL) beamline 4-3 with an unfocused beam. Second-order harmonics were rejected using a Pt-coated mirror.

\section{GRAZING INCIDENCE SPECTROMETER}

The grazing incidence spectrometer has been developed at the Biotechnology Laboratory of SSRL to be used for grazing incidence XAS and XSW measurements. Details of the spectrometer will be presented in a future publication (IJP/RM, manuscript in preparation). The spectrometer includes an optical rail to align to the x-ray beam, motorized collimating slits in both the vertical and horizontal directions, a vertical alignment to position the sample precisely in the beam and a $\theta$ axis to scan the angle of incidence. All of these axes are driven by stepper-motors and are under computer control. The alignment procedure for XSW involved first setting the slits to the required aperture, to allow for complete interception of the beam by the sample at all angles of interest, then aligning the optical rail so that the x-ray beam was maximized in the center of collimating slits both before and after the sample. After this, the gold mirror substrate with deposited OEC oriented monolayer was mounted on the $\theta$ stage and both the vertical position of the sample and the zero-point of the $\theta$ angle were checked by scanning the respective motors. During the XSW measurements, the angle $\theta$ was scanned while simultaneously monitoring the reflected signal using a nitrogen-filled ion chamber, and the fluorescence signals using a Canberra 13-element germanium $\mathrm{x}$-ray fluorescence detector.

\section{RESULTS}

To investigate the feasibility of detecting Mn signals from a dilute sample of OEC, MCA counts were taken on a sample equivalent to 300 monolayers (188 seconds of collection time) and compared with that of a protein-free thiol-Au sample collected over 271 seconds (Fig. 2). Clearly, there

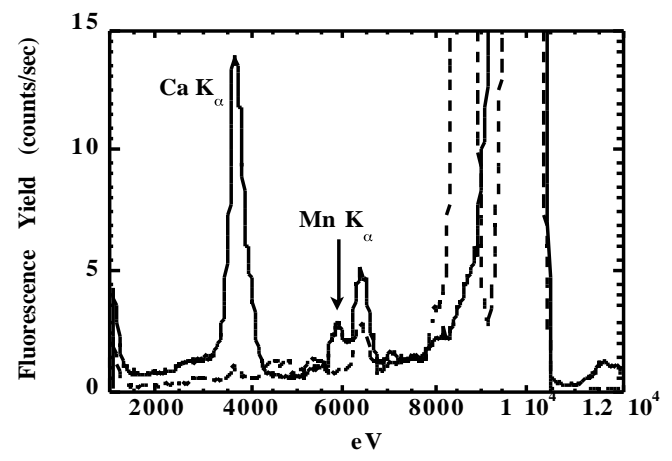

FIG. 2. Normalized MCA data collected for thiol-coated Au (dashed line), 300 monolayer equivalents of OEC on thiol-Au (solid line).

is a significant $\mathrm{Mn}$ signal present in the 300 monolayer sample that is absent in the thiol-Au control sample. We estimated the Mn signal-to-noise ratio to be 95:1, a factor of approximately 20-fold greater than that required for statistically meaningful data acquisition. This result suggests that we should be able to collect a complete $\theta$ scan with statistically significant Mn signal in approximately 5 hours from a sample that is 300 times as dilute, such as a monolayer of OEC.

A number of OEC monolayer samples were measured initially with the MCA. It was noted that the ratio of Ca-toMn fluorescence is significantly greater than that expected from stoichiometric considerations alone. The inclusion of an additional rinsing step after drying the sample seems to 
remove the excess $\mathrm{Ca}$ and restore the $\mathrm{Ca}: \mathrm{Mn}$ ratio to a reasonable value for an OEC monolayer. Figure 3a shows the MCA data from two monolayer samples of OEC prepared as described in Section III, before and after re-rinsing. Incubation of the thiol-Au substrate in suspensions of OEC that lacked the detergent DM had the same effect of preventing Ca contamination (Fig. 3b).

Monolayer Mn XSW fluorescence could not be obtained using these samples due to $\mathrm{Cr}$ contamination in the experiment. There is a statistically significant amount of $\mathrm{Cr}$ present, such that the fluorescence yield of the $\mathrm{Cr} \mathrm{K}_{\beta}$ emission overwhelms the $\mathrm{Mn} \mathrm{K}_{\alpha}$ signal. This problem is also illustrated in Figure $3 \mathrm{~b}$. While we have not located the exact source of the $\mathrm{Cr}$ contamination, progress is being made to minimize $\mathrm{Cr}$ in the sample environment.
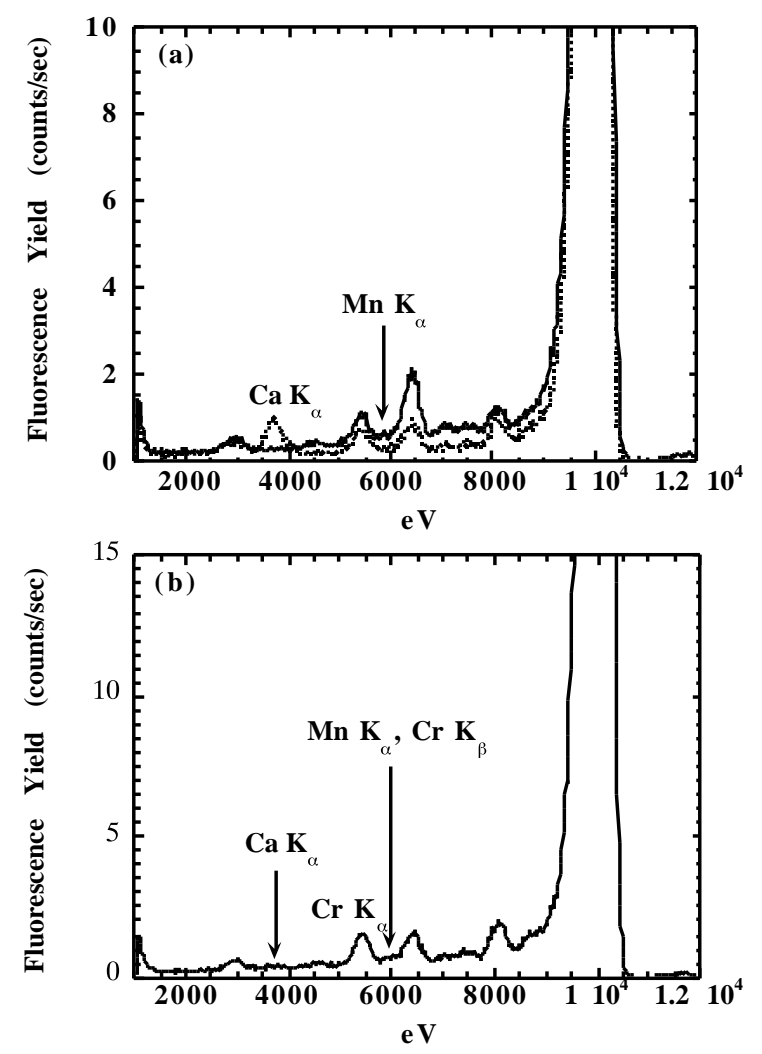

FIG. 3. Normalized MCA data collected for (a) monolayer prepared in solubilized OEC without re-rinsing to remove the excess $\mathrm{Ca}^{2+}$ (dotted line) and re-rinsed (solid line); (b) monolayer prepared in suspension of OEC without DM. A significant amount of $\mathrm{Cr}$ is present in all samples.

In contrast, $\mathrm{Ca}$ XSW measurements were straightforward, as shown in Figure 4. The oscillations in the Ca XSW fluorescence yield suggests that the $\mathrm{Ca}^{2+}$ ions are located at a well defined distance from the surface of the thiol-Au mirror, demonstrating that we may have serendipitously fabricated a protein sample coated with a layer of $\mathrm{Ca}^{2+}$ ions, which can be removed by rinsing with Ca-free buffer. There are nearly two full oscillations in the Ca fluorescence yield before the critical angle is reached, which places the Ca atoms at roughly $115 \AA$ from the mirror surface. This distance would be consistent with sample thicknesses as determined by ellipsometry. However, the uncertainties in this preliminary data do not permit unambiguous location of the $\mathrm{Ca}^{2+}$ ions. Regardless of the molecular basis of the Ca deposition, it is noteworthy that clear oscillations in the Ca fluorescence yield can be seen in a sample whose $\mathrm{Mn}$ and $\mathrm{Ca}$ contents are comparable (Fig. 3a).

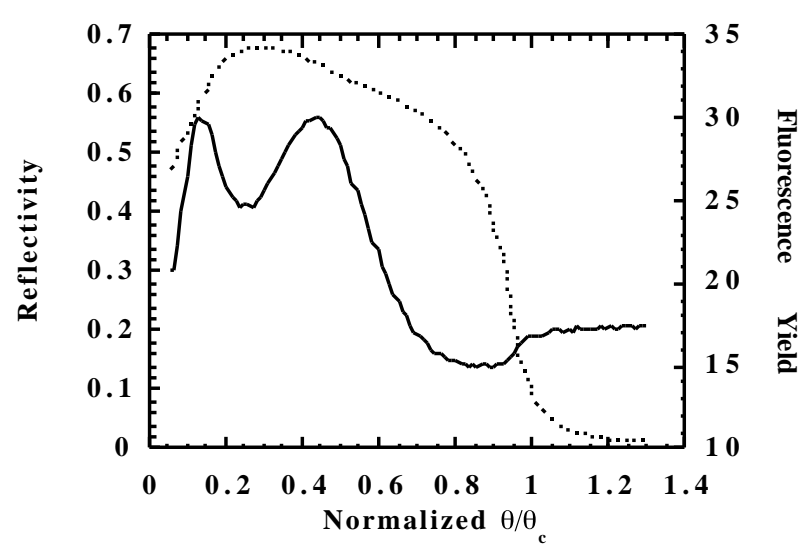

FIG. 4. Reflectivity (dotted line) and Ca XSW fluorescence (solid line) of OEC monolayer with excess Ca present. These data were collected with a step size of $0.087 \mathrm{mrad}$ at $100 \mathrm{secs}$ per point and signal-averaged over 9 scans.

\section{CONCLUSION}

It was demonstrated in this feasibility study that it is possible to obtain Mn XSW fluorescence data with good signal-to-noise ratio for monolayer samples of OEC. Based on these measurements, the necessary scan times will be approximately 5 hours for measurements of Mn XSW once the $\mathrm{Cr}$ contamination has been removed.

\section{ACKNOWLEDGMENT}

Supported in part by the NIH (GM-45205 to JEPH) and the USDA (92-37306-7662 to CFY). SSRL is funded by the Department of Energy, Office of Basic Energy Sciences. The Biotechnology Program at SSRL is supported by the NIH, Biomedical Research Technology Program, Division of Research Resources. Further support is provided by the Department of Energy, Office of Health and Environmental Research. We thank Prof. Martin Caffrey and Dr. Jin Wang (The Ohio State University) for assistance in the early stages of this project.

1 B.W. Batterman, "Effect of dynamical diffraction in x-ray fluorescence scattering," Phys. Rev. 133, 759-64 (1964).

2 T.W. Barbee, W.K. Warburton, and J.H. Underwood, "Determination of the x-ray anomalous dispersion of titanium made with a titanium-carbon layered synthetic microstructure," J. Opt. Soc. Am. B 1, 691-8 (1984).

3 M.J. Bedzyk, D.H. Bilderback, G.M. Bommarito et al., "X-ray Standing Waves: A Molecular Yardstick for Biological Membranes," Science 241, 1788-91 (1988).

4 M. Caffrey and J. Wang, "Internal and Interfacial Structure of Membranes studied using X-Ray Standing Waves," Faraday Discuss. Chem. Soc. 94, 283-93 (1992). 
5 J. Wang, C.J.A. Wallace, I. Clark-Lewis et al., "Structure Characterization of Membrane Bound and Surface Adsorbed Protein," J. Mol. Biol. 237, 1-4 (1994).

6 R.J. Debus, "The manganese and calcium ions of photosynthetic oxygen evolution," Biochimica et Biophysica Acta 1102, 269-352 (1992).
7 M.J. Bedzyk, G.M. Bommarito, and J.S. Schildkraut, "X-Ray Standing Waves at a Reflecting Mirror Surface," Phys. Rev. Lett. 62 (12), 1376-1379 (1989).

8 J. Wang, M.J. Bedzyk, T. Penner et al., "Structural studies of membrane and surface layers up to $1,000 \AA$ thick using X-ray standing waves," Nature (London) 354, 377-80 (1991). 